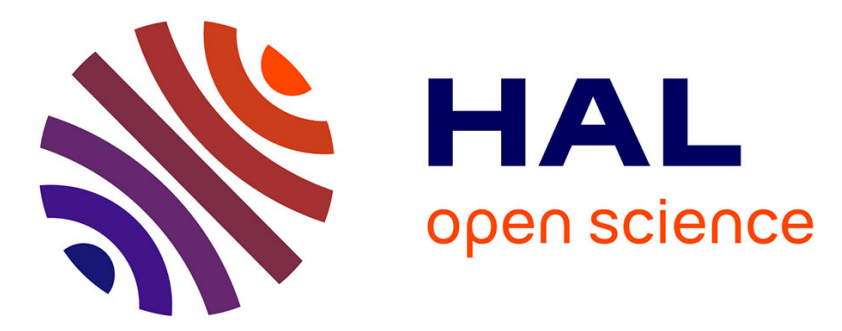

\title{
Low-rank space-time decoupled isogeometric analysis for parabolic problems with varying coefficients
}

Angelos Mantzaflaris, Felix Scholz, Ioannis Toulopoulos

\section{To cite this version:}

Angelos Mantzaflaris, Felix Scholz, Ioannis Toulopoulos. Low-rank space-time decoupled isogeometric analysis for parabolic problems with varying coefficients. Computational Methods in Applied Mathematics, 2019, 19 (1), pp.123-136. 10.1515/cmam-2018-0024 . hal-02271808

\section{HAL Id: hal-02271808 \\ https://hal.inria.fr/hal-02271808}

Submitted on 27 Aug 2019

HAL is a multi-disciplinary open access archive for the deposit and dissemination of scientific research documents, whether they are published or not. The documents may come from teaching and research institutions in France or abroad, or from public or private research centers.
L'archive ouverte pluridisciplinaire HAL, est destinée au dépôt et à la diffusion de documents scientifiques de niveau recherche, publiés ou non, émanant des établissements d'enseignement et de recherche français ou étrangers, des laboratoires publics ou privés. 


\title{
Low-rank space-time decoupled isogeometric analysis for parabolic problems with varying coefficients
}

\begin{abstract}
In this paper we present a space-time isogeometric analysis scheme for the discretization of parabolic evolution equations with diffusion coefficients depending on both time and space variables. The problem is considered in a space-time cylinder in $\mathbb{R}^{d+1}$, with $d=2,3$ and is discretized using higher-order and highly-smooth spline spaces. This makes the matrix formation task very challenging from a computational point of view. We overcome this problem by introducing a low-rank decoupling of the operator into space and time components. Numerical experiments demonstrate the efficiency of this approach.
\end{abstract}

Keywords: Isogeometric Analysis, low-rank approximation, parabolic initial-boundary value problems, B-splines, isogeometric matrix assembly

\section{Introduction}

In this work we apply an efficient Isogeometric Analysis $(\operatorname{IgA})$ scheme $[1,8]$ to parabolic initial-boundary value problems, which are frequently used to describe time evolution phenomena in physics, medicine, and so on.

The standard approach for these problems is to discretize separately in space and in time. For high dimensions, there are works which propose tensor methods in order to tackle the, so called, curse of dimensionality, notably using the tensor-train format for a global space-time approximation $[4,5]$. Recently, it was proposed in [13] to use IgA to discretize a pure heat conduction problem simultaneously in both space and time. In particular, the time variable was regarded as an extra spatial variable, and the problem was lifted in one dimension higher. Consequently, the space-time cylinder was parameterized by a NURBS volume and high-order and highly smooth splines were used to discretize the problem.

One issue in the efficiency of $\mathrm{IgA}$ is the increased cost of computations already for 3D problems. When adding an extra dimension, the cost related to computing the discretized operator increases significantly [15]. Indeed, the dependence of the computational complexity with respect to the dimension is exponential [14] In the recent work [17] a partial low-rank tensor decomposition was proposed for decoupling the integrals arising in isogeometric schemes, thereby accelerating their computation.

The present work combines [13] and [17]. In particular, the scheme used in [13] is applied to a general parabolic problem with varying diffusion coefficient. We revisit the analysis in [13] for the case in question, and provide the corresponding discretization error estimate in the appropriate norm. Moreover, the fully varying coefficient requires highly accurate numerical integration in $\mathbb{R}^{d+1}$, which becomes practically infeasible, even for a small or moderate number of degrees of freedom. In order to treat this problem efficiently, we use the low-rank decoupling techniques proposed in [17]. This provides us with an efficient Kronecker decomposition of the system matrix into space and time components, therefore reducing the dimension of the problem as well as the overall computational effort.

The IgA space-time scheme in [13] is based on the space-time variational formulation presented in $[10,11]$. In these works, the authors proved the uniqueness of the corresponding weak solution. Working in a different

Angelos Mantzaflaris, Felix Scholz, loannis Toulopoulos, Johann Radon Institute for Computational and Applied Mathematics (RICAM), Austrian Academy of Sciences, Altenberger Straße 69, A-4040 Linz, Austria 
direction, in [7] a time discontinuous Galerkin space-time IgA scheme has been analyzed for solving simple parabolic problems (i.e., without varying coefficients).

The rest of the paper is organized as follows. We start by introducing our model problem, deriving the discrete variational form and the error estimates for spline discretizations in Section 2. In Section 3 we focus on the efficient computation of the matrix expressing the discrete operator and we bound the computational complexity in terms of the number of degrees of freedom and the polynomial degree of the discretization. We provide numerical results and computation times in Section 4. We conclude the paper and provide some future research directions in Section 5.

\section{The model problem}

\subsection{Preliminaries}

Let $\Omega$ be a bounded Lipschitz domain in $\mathbb{R}^{d}$, with some integer $d \geq 1$. From a practical point of view, we are interested in the cases $d=1,2$, or 3. Furthermore, $\boldsymbol{\alpha}=\left(\alpha_{1}, \ldots, \alpha_{d}\right)$ denotes a multi-index of non-negative integers $\alpha_{1}, \ldots, \alpha_{d}$ with degree $|\boldsymbol{\alpha}|=\sum_{j=1}^{d} \alpha_{j}$. For any $\boldsymbol{\alpha}$, we define the differential operator $\partial_{x}^{\boldsymbol{\alpha}}=\partial_{x_{1}}^{\alpha_{1}} \ldots \partial_{x_{d}}^{\alpha_{d}}$, with $\partial_{x_{j}}=\partial / \partial x_{j}, j=1, \ldots, d$. As usual, $L^{2}(\Omega)$ denotes the Lebesgue space for which $\int_{\Omega}|v(x)|^{2} d x<\infty$, endowed with the norm $\|v\|_{L^{2}(\Omega)}=\left(\int_{\Omega}|v(x)|^{2} d x\right)^{\frac{1}{2}}$. For an integer $\ell \geq 0$, we define the standard Sobolev space

$$
H^{\ell}(\Omega)=\left\{v \in L^{2}(\Omega): \partial_{x}^{\boldsymbol{\alpha}} v \in L^{2}(\Omega), \text { for all }|\boldsymbol{\alpha}| \leq \ell\right\},
$$

endowed with the norm

$$
\|v\|_{H^{\ell}(\Omega)}=\left(\sum_{|\boldsymbol{\alpha}| \leq \ell}\left\|\partial_{x}^{\boldsymbol{\alpha}} v\right\|_{L^{2}(\Omega)}^{2}\right)^{\frac{1}{2}}
$$

and also, we introduce the subspace $H_{0}^{1}(\Omega)=\left\{v \in H^{1}(\Omega): v=0\right.$ on $\left.\partial \Omega\right\}$. Let $\bar{J}=[0, T]$ be the time interval for some final time $T>0$. For later use, we define the space-time cylinder $Q=\Omega \times(0, T)$ and its boundary parts $\Sigma=\partial \Omega \times(0, T), \Sigma_{T}=\Omega \times\{T\}$ and $\Sigma_{0}=\Omega \times\{0\}$, such that $\partial Q=\Sigma \cup \bar{\Sigma}_{0} \cup \bar{\Sigma}_{T}$, see an illustration in Fig. 1.

According to the definition of $\partial_{x}^{\alpha}$, we now define the spatial gradient $\nabla_{x} v=\left(\partial_{x_{1}} v, \ldots, \partial_{x_{d}} v\right)$. Let $\ell$ and $m$ be positive integers. For functions defined in the space-time cylinder $Q$, we define the Sobolev spaces

$$
H^{\ell, m}(Q)=\left\{v \in L_{2}(Q): \partial_{x}^{\boldsymbol{\alpha}} v \in L_{2}(Q), 0 \leq|\boldsymbol{\alpha}| \leq \ell \text { and } \partial_{t}^{i} v \in L_{2}(Q), i=1, \ldots, m\right\},
$$

where $\partial_{t}=\partial / \partial t$, and, in particular, the subspaces

$$
\begin{aligned}
& H_{0}^{1,0}(Q)=\left\{v \in L_{2}(Q): \nabla_{x} v \in\left[L_{2}(Q)\right]^{d}, v=0 \text { on } \Sigma\right\} \text { and } \\
& H_{0, \overline{0}}^{1,1}(Q)=\left\{v \in L_{2}(Q): \nabla_{x} v \in\left[L_{2}(Q)\right]^{d}, \partial_{t} v \in L_{2}(Q), v=0 \text { on } \Sigma, v=0 \text { on } \Sigma_{T}\right\}, \\
& H_{0, \underline{0}}^{1,1}(Q)=\left\{v \in L_{2}(Q): \nabla_{x} v \in\left[L_{2}(Q)\right]^{d}, \partial_{t} v \in L_{2}(Q), v=0 \text { on } \Sigma, v=0 \text { on } \Sigma_{0}\right\} .
\end{aligned}
$$

We equip the above spaces with the norms and seminorms

$$
\begin{aligned}
\|v\|_{H^{\ell, m}(Q)} & =\left(\sum_{|\boldsymbol{\alpha}| \leq \ell}\left\|\partial_{x}^{\boldsymbol{\alpha}} v\right\|_{L^{2}(Q)}^{2}+\sum_{m_{0}=0}^{m}\left\|\partial_{t}^{m_{0}} v\right\|_{L^{2}(Q)}^{2}\right)^{\frac{1}{2}} \\
|v|_{H^{\ell, m}(Q)} & =\left(\sum_{|\boldsymbol{\alpha}|=\ell}\left\|\partial_{x}^{\boldsymbol{\alpha}} v\right\|_{L^{2}(Q)}^{2}+\left\|\partial_{t}^{m} v\right\|_{L^{2}(Q)}^{2}\right)^{\frac{1}{2}} .
\end{aligned}
$$

respectively. In what follows, positive constants $c$ and $C$ appearing in inequalities are generic constants which do not depend on the mesh-size $h$. We write $a \sim b$ meaning that $c a \leq b \leq C a$ with generic positive constants $c$ and $C$. 


\subsection{The model parabolic problem}

In $\bar{Q}=\bar{\Omega} \times[0, T]$, we consider the initial boundary value problem

$$
\begin{aligned}
\partial_{t} u-\operatorname{div}_{x}(\rho(x, t) \nabla u) & =f \text { in } Q \text { and } \\
u(x, t) & =0 \text { on } \Sigma, \quad u(\cdot, 0)=u_{0} \text { on } \Sigma_{0},
\end{aligned}
$$

as model problem, where $f: Q \rightarrow \mathbb{R}$, with $f \in L^{2}(Q)$, and $u_{0}: \Omega \rightarrow \mathbb{R}$, with $u_{0} \in L^{2}(\Omega)$ are given functions, the diffusion coefficient $\rho_{\max } \geq \rho(x, t) \geq \rho_{\min }>0$ is a given smooth function, and $u: \bar{Q} \rightarrow \mathbb{R}$ is the unknown.

Using the standard procedure and integration by parts with respect to both $x$ and $t$, we can easily derive the following space-time variational formulation of (2): find $u \in H_{0}^{1,0}(Q)$ such that

$$
a(u, v)=l(v), \quad \text { for all } v \in H_{0, \overline{0}}^{1,1}(Q)
$$

with the bilinear form

$$
a(u, v)=-\int_{Q} u(x, t) \partial_{t} v(x, t) d x d t+\int_{Q} \rho(x, t) \nabla_{x} u(x, t) \cdot \nabla_{x} v(x, t) d x d t
$$

and the linear form

$$
l(v)=\int_{Q} f(x, t) v(x, t) d x d t+\int_{\Omega} u_{0}(x) v(x, 0) d x,
$$

where note that the last integral in (5) is related to the initial conditions in (2). For simplicity, we only consider homogeneous Dirichlet boundary conditions on $\Sigma$. Also, in the rest of the paper, we will consider that $u_{0}=0$. However, the analysis presented in the sequel can easily be generalized to other constellations of boundary conditions. The space-time variational formulation (3) has a unique solution, see, e.g, [10, 11]. In these monographs, besides existence and uniqueness results, one can also find useful a priori estimates and regularity results.

For our analysis, we make the following convenient assumption.

Assumption 2.1. We assume that the solution $u$ of (3) belongs to $V=H_{0, \underline{0}}^{1,1}(Q) \cap H^{\ell, m}(Q)$ with some $\ell \geq 2$ and $m \geq 1$.

\subsection{B-spline spaces}

In this section, we briefly present the B-spline spaces and the form of the B-spline parameterizations for the physical space-time patches (called also space-time subdomains). We refer to $[1,3]$, for a more detailed presentation.

We start by presenting the B-spline space for the univariate case. Let the integer $p$ denote the B-spline degree and the integer $n_{1}$ denote the number of basis functions. Consider a partition $\mathcal{Z}=\left\{0=z_{1}<z_{2}<\right.$ $\left.\cdots<z_{M}=1\right\}$ of $\bar{I}=[0,1]$ with $\bar{I}_{j}=\left[z_{j}, z_{j+1}\right], j=1, \ldots, M-1$ being the intervals of the partition. Based on $\mathcal{Z}$, we consider a knot-vector $\Xi=\left\{0=\xi_{1}, \xi_{2}, \ldots, \xi_{n_{1}+p+1}=1\right\}$ and its associated vector of knot multiplicities $\mathcal{M}=\left\{m_{1}, \ldots, m_{M}\right\}$ with $m_{1}=m_{M}=p+1$, i.e.,

$$
\xi_{1+m_{1}+\cdots+m_{j-1}}=\cdots=\xi_{m_{1}+\cdots+m_{j}}=z_{j} \quad \text { for } \quad j=1, \ldots, M .
$$

We assume that $m_{j} \leq p$ for all internal knots. The B-spline basis functions are defined by the Cox-de Boor formula, see, e.g., [1] and [3],

$$
\hat{B}_{i, p}=\frac{x-\xi_{i}}{\xi_{i+p}-\xi_{i}} \hat{B}_{i, p-1}(x)+\frac{\xi_{i+p+1}-x}{\xi_{i+p+1}-\xi_{i+1}} \hat{B}_{i+1, p-1}(x),
$$

with $\hat{B}_{i, 0}(x)= \begin{cases}1, & \text { if } \xi_{i} \leq x \leq \xi_{i+1}, \\ 0, & \text { otherwise. }\end{cases}$ 
Now, let us consider the unit cube $\widehat{Q}=(0,1)^{d+1} \subset \mathbb{R}^{d+1}$, which we will refer to as the parametric domain. We extend the univariate B-spline concept to multiple dimensions with the use of tensor products. Let the integers $p$ and $n_{k}$ denote the given B-spline degree (same for all directions) and the number of basis functions of the B-spline space that will be constructed in $x_{k}$-direction with $k=1, \ldots, d+1$. We introduce the $(d+1)$-dimensional vector of knots $\Xi^{d+1}=\left(\Xi^{1}, \ldots, \Xi^{k}, \ldots, \Xi^{d+1}\right), k=1, \ldots, d+1$, with the particular components given by $\Xi^{k}=\left\{0=\xi_{1}^{k}, \xi_{2}^{k}, \ldots, \xi_{n_{k}+p+1}^{k}=1\right\}$. For all the internal knots, we assume that $m_{j}^{k} \leq p$, with $m_{j}^{k}$ to be the associated multiplicities. The basis functions of the multivariate B-spline space $\hat{\mathbb{B}}_{\Xi^{d+1}, p}$ are defined by the tensor-product of the corresponding univariate B-spline basis functions of $\hat{\mathbb{B}}_{\Xi^{k}, p}$ spaces, that is

$$
\hat{\mathbb{B}}_{\Xi^{d+1}, p}=\bigotimes_{k=1}^{d+1} \hat{\mathbb{B}}_{\Xi^{k}, p}=\operatorname{span}\left\{\hat{B}_{j}(\hat{x})\right\}_{j=1}^{n_{B}},
$$

where $n_{B}=n_{1} \cdots n_{d+1}$ and each $\hat{B}_{j}(\hat{x})$ has the form

$$
\hat{B}_{j}(\hat{x})=\hat{B}_{j_{1}}\left(\hat{x}_{1}\right) \cdots \hat{B}_{j_{k}}\left(\hat{x}_{k}\right) \cdots \hat{B}_{j_{d+1}}\left(\hat{x}_{d+1}\right), \text { with } \hat{B}_{j_{k}}\left(\hat{x}_{k}\right) \in \hat{\mathbb{B}}_{\Xi^{k}, p} .
$$

In the frame of $\operatorname{IgA}$, the representation of any volumetric domain is defined by a B-spline basis, see (7), and the associated control points, see [1]. Given the associated control points $\mathbf{C}_{j} \in \mathbb{R}^{d+1}$, the domain $Q$ is parameterized by the mapping

$$
\boldsymbol{\Phi}: \widehat{Q} \rightarrow Q, \quad x=\boldsymbol{\Phi}(\hat{x})=\sum_{j=1}^{n_{B}} \mathbf{C}_{j} \hat{B}_{j}(\hat{x}) \in Q,
$$

where $\hat{x}=\boldsymbol{\Phi}^{-1}(x)$. cf. [1]. The components $\Xi^{k}$ of $\boldsymbol{\Xi}^{d+1}$ form a mesh $T_{\hat{h}, \widehat{Q}}=\left\{\hat{E}_{m}\right\}_{m=1}^{M}$ in $\widehat{Q}$, where $\hat{E}_{m}$ are the elements and $\hat{h}$ is the mesh size. We construct a mesh $T_{h, Q}=\left\{E_{m}\right\}_{m=1}^{M}$ in $Q$, where the elements $E_{m}$ are the images of $\hat{E}_{m} \in T_{\hat{h}, \widehat{Q}}$ under $\boldsymbol{\Phi}$. We define the isogeometric discretization space on $Q$ as

$$
V_{\Phi, \Xi^{d+1}, p}:=\operatorname{span}\left\{\hat{B}_{j} \circ \Phi^{-1}: \hat{B}_{j} \in \hat{\mathbb{B}}_{\Xi^{d+1}, p}, j=1, \ldots, n_{B}\right\} .
$$

To keep notation simple, we denote the above space by $V_{h}$, that is, we omit to write the domain parameterization, the spline degree and knot vectors. Furthermore, we introduce the space

$$
V_{0 h}:=V_{h} \cap H_{0, \underline{0}}^{1,1}=\left\{v_{h} \in V_{h}:\left.v_{h}\right|_{\Sigma \cup \Sigma_{0}}=0\right\} .
$$

Assumption 2.2. The mesh $T_{\hat{h}, \widehat{Q}}$ is uniform, i.e., for every $\hat{E} \in T_{\hat{h}, \widehat{Q}}$, there exist a number $\gamma>0$ such that $\gamma \leq \hat{h} / r_{\hat{E}}$, where $r_{\hat{E}}$ is the radius of the inscribed circle of $\hat{E}$.

Remark 1. Since the parameterization $\boldsymbol{\Phi}$, is fixed, under the Assumption 2.2, we have that $h \sim \hat{h}$.

The parameterization $\boldsymbol{\Phi}$, can be considered to be bi-Lipschitz homeomorphisms, [2]. For simplifying the analysis, we consider the following regularity properties on $\boldsymbol{\Phi}$.

Assumption 2.3. We assume that $\boldsymbol{\Phi}$ and $\boldsymbol{\Phi}^{-1}$ are sufficiently smooth (i.e., $C^{1}$ diffeomorphisms) and that there exist constants $0<c<C$ such that $c \leq\left|\operatorname{det} J_{\boldsymbol{\Phi}}\right| \leq C$, where $J_{\boldsymbol{\Phi}}$ is the Jacobian matrix of $\boldsymbol{\Phi}$.

\subsection{Discrete variational forms}

We denote by $\mathbf{n}=\left(n_{1}, \cdots, n_{d}, n_{d+1}\right)=\left(n_{x}, n_{t}\right)$ the normal on $\Sigma$. Let $v_{h} \in V_{0 h}$ and $w_{h}^{n}=v_{h}+\theta h \partial_{t} v_{h}$, where $\theta$ is a positive parameter. We multiply (2) by $w_{h}$ and we integrate over $Q$; by applying integration by 


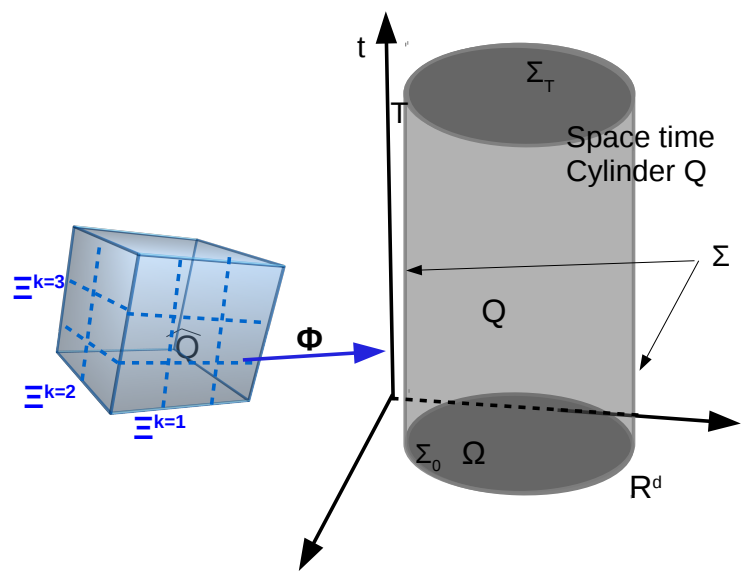

Fig. 1: The space type cylinder $Q$ and the $\operatorname{IgA}$ B-spline parameterization $\boldsymbol{\Phi}: \hat{Q} \rightarrow Q$.

parts we obtain

$$
\begin{gathered}
a_{Q}\left(u, v_{h}\right)=\int_{Q} \partial_{t} u\left(v_{h}+\theta h \partial_{t} v_{h}\right)+\rho(x, t)\left(\nabla_{x} u \cdot \nabla_{x} v_{h}+\theta h \nabla_{x} u \cdot \nabla_{x} \partial_{t} v_{h}\right) d x d t \\
+\int_{\partial Q} n_{x} \cdot \rho(x, t) \nabla_{x} u\left(v_{h}+\theta h \partial_{t} v_{h}\right) d s=\int_{Q} f\left(v_{h}+\theta h \partial_{t} v_{h}\right) d x d t
\end{gathered}
$$

Since $w_{h}=0$ on $\Sigma$, and $n_{x}=0$ on $\Sigma_{0} \cup \Sigma_{T}$, we can obtain

$$
\begin{aligned}
a_{Q}\left(u, v_{h}\right) & =\int_{Q} \partial_{t} u\left(v_{h}+\theta h \partial_{t} v_{h}\right)+\rho(x, t)\left(\nabla_{x} u \cdot \nabla_{x} v_{h}+\theta h \nabla_{x} u \cdot \nabla_{x} \partial_{t} v_{h}\right) d x d t \\
& =\int_{Q} f\left(v_{h}+\theta h \partial_{t} v_{h}\right) d x d t .
\end{aligned}
$$

The space-time IgA method for (2) can be formulated as follows: Find $u_{h} \in V_{0 h}$ such that

$$
a_{h}\left(u_{h}, v_{h}\right)=L_{h}\left(v_{h}\right), \quad \forall v_{h} \in V_{0 h}
$$

where

$$
a_{h}\left(u_{h}, v_{h}\right)=a_{Q}\left(u_{h}, v_{h}\right) \text { and } L_{h}\left(v_{h}\right)=\int_{Q} f\left(v_{h}+\theta h \partial_{t} v_{h}\right) d x d t .
$$

Note that, under the Assumption 2.1 and the derivation of (13), we can conclude that the solution $u$ of (3) satisfies (14a).

\subsubsection{Discretization error analysis}

For simplifying the presentation, we derive the analysis for the case where $\rho(x, t)$ is constant, i. e., $\rho(x, t)=$ $\rho>0$. The analysis can be easily extended to the general problem given in (2). Motivated by (14b), we define the norm on $V_{0 h}$

$$
\|v\|_{h}=\left(\left\|\rho^{\frac{1}{2}} \nabla_{x} v\right\|_{L^{2}(Q)}^{2}+\theta h\left\|\partial_{t} v\right\|_{L^{2}(Q)}^{2}+\frac{1}{2}\|v\|_{L^{2}\left(\Sigma_{T}\right)}^{2}\right)^{\frac{1}{2}}
$$

Let the Assumption 2.1 and the space $V$ defined there. We define the space $V_{0 h, *}=V+V_{0 h}$ endowed with the norm

$$
\|v\|_{h, *}=\left(\|v\|_{h}^{2}+(\theta h)^{-1}\|v\|_{L^{2}(Q)}^{2}\right)^{\frac{1}{2}} .
$$


Lemma 2. The discrete bilinear form $a_{h}(\cdot, \cdot)$, defined in (14a), is $V_{0 h}$-elliptic, i.e.,

$$
a_{h}\left(v_{h}, v_{h}\right) \geq C_{e}\left\|v_{h}\right\|_{h}^{2}, \quad \text { for } v_{h} \in V_{0 h},
$$

where $C_{e}=1$.

Proof. Using Green's formula $\int_{Q} \partial_{t} v_{h} v_{h}+v_{h} \partial_{t} v_{h} d x d t=\int_{\partial Q} n_{t} v_{h}^{2} d s$, the fact that $n_{t}=0$ on $\Sigma$ and $v_{h}(x, 0)=0$, we obtain the identity

$$
\int_{Q} \partial_{t} v_{h} v_{h}=\frac{1}{2} \int_{Q} \partial_{t} v_{h}^{2} d x d t=\frac{1}{2} \int_{\Sigma_{T}} v_{h}^{2} d s
$$

The definition of $a_{h}(\cdot, \cdot)$ and identity (18) yield

$$
\begin{aligned}
a_{h}\left(v_{h}, v_{h}\right)= & \int_{Q} \frac{1}{2} \partial_{t} v_{h}^{2}+\theta h\left(\partial_{t} v_{h}\right)^{2}+\rho\left|\nabla_{x} v_{h}\right|^{2}+\rho \frac{\theta h}{2} \partial_{t}\left|\nabla_{x} v_{h}\right|^{2} d x d t \\
= & \frac{1}{2}\left\|v_{h}\right\|_{L^{2}\left(\Sigma_{T}\right)}^{2}+\theta h\left\|\partial_{t} v_{h}\right\|_{L_{2}(Q)}^{2}+\left\|\rho^{\frac{1}{2}} \nabla_{x} v_{h}\right\|_{L_{2}(Q)}^{2}+\frac{\theta h}{2} \int_{\partial Q} \rho\left|\nabla_{x} v_{h}\right|^{2} n_{t} d s \\
= & \frac{1}{2}\left\|v_{h}\right\|_{L^{2}\left(\Sigma_{T}\right)}^{2}+\theta h\left\|\partial_{t} v_{h}\right\|_{L_{2}(Q)}^{2}+\left\|\rho^{\frac{1}{2}} \nabla_{x} v_{h}\right\|_{L_{2}(Q)}^{2} \\
& \quad+\frac{\theta h}{2}\left(\left\|\rho^{\frac{1}{2}} \nabla_{x} v_{h}\right\|_{L_{2}\left(\Sigma_{T}\right)}^{2}-\left\|\rho^{\frac{1}{2}} \nabla_{x} v_{h}\right\|_{L_{2}\left(\Sigma_{0}\right)}^{2}\right) .
\end{aligned}
$$

Now, since it holds that $v_{h}(x, 0)=0$, we also get $\nabla_{x} v_{h}(x, 0)=0$ for all $x \in \Sigma_{0}$. Using this in (19), we can arrive at

$$
\begin{aligned}
a_{h}\left(v_{h}, v_{h}\right)= & \frac{1}{2}\left\|v_{h}\right\|_{L^{2}\left(\Sigma_{T}\right)}^{2}+\theta h\left\|\partial_{t} v_{h}\right\|_{L_{2}(Q)}^{2} \\
& +\left\|\rho^{\frac{1}{2}} \nabla_{x} v_{h}\right\|_{L_{2}(Q)}^{2}+\frac{\theta h}{2}\left\|\rho^{\frac{1}{2}} \nabla_{x} v_{h}\right\|_{L_{2}\left(\Sigma_{T}\right)}^{2} \geq\left\|v_{h}\right\|_{h}^{2} .
\end{aligned}
$$

Lemma 3. The discrete bilinear form $a_{h}(\cdot, \cdot)$, is uniformly bounded on $V_{0 h, *} \times V_{0 h}$, i.e.,

$$
\left|a_{h}\left(u, v_{h}\right)\right| \leq \mu_{b}\|u\|_{h, *}\left\|v_{h}\right\|_{h}, \quad \text { for } u \in V_{0 h, *} \text { and } v_{h} \in V_{0 h},
$$

where the constant $\mu_{b}>0$ depends on the constants that appear in the inverse inequalities.

Proof. The proof can be given following the same steps as in Lemma 4 in [13].

Lemma 4. Let $s \geq 2$ be a positive integer and let a function $v \in V=H^{s}(Q) \cap H_{0, \underline{0}}^{1,1}(Q)$. Then there exists a tensor-product quasi-interpolant $\Pi_{h, p}: V \rightarrow V_{0 h}$ such that

$$
\begin{aligned}
\left\|u-\Pi_{h, p} u\right\|_{h} & \leq C_{\mathrm{intp}, 1} h^{\min (p+1, s)-1}\|u\|_{H^{s}(Q)}, \\
\left\|u-\Pi_{h, p} u\right\|_{h, *} & \leq C_{\mathrm{intp}, 2} h^{\min (p+1, s)-1}\|u\|_{H^{s}(Q)},
\end{aligned}
$$

where the constants $C_{\mathrm{intp}, i}, i=1,2$ depend on the B-spline parameterization $\mathbf{\Phi}$, but not on $u$ and $h$.

Proof. The proof can be given using the classical inverse and trace inequalities and using the quasiinterpolation estimates on B-spline spaces presented in [2]. See also Lemma 6 in [13].

Now, we can give the main discretization error estimate in terms of the discrete norm $\|\cdot\|_{h}$. 
Theorem 5. Let $u \in V=H^{s}(Q) \cap H_{0, \underline{0}}^{1,1}(Q)$, with $s \geq 2$ solve (3) and $u_{h}$ solve (14a). Under Assumption 2.2, there exist a $c>0$, independent of $\bar{u}$ and $h$ such that

$$
\left\|u-u_{h}\right\|_{h} \leq \operatorname{ch}^{r}\|u\|_{H^{s}(\Omega)}, \quad \text { with } \quad r=\min (p+1, s)-1 .
$$

Proof. Using the properties of bilinear form $a_{h}(\cdot, \cdot)$, i.e., Lemma 2 and Lemma 3, as well as the consistency of $u$, we obtain

$$
\begin{array}{r}
\left\|u_{h}-\Pi_{h} u\right\|_{h}^{2} \leq C_{e} a_{h}\left(u_{h}-\Pi_{h, p} u, u_{h}-\Pi_{h, p} u\right) \\
\quad=a_{h}\left(u-\Pi_{h, p} u, u_{h}-\Pi_{h, p} u\right) \leq \mu_{b}\left\|u-\Pi_{h} u\right\|_{h, *}\left\|u_{h}-\Pi_{h} u\right\|_{h},
\end{array}
$$

where we immediately get $\left\|u_{h}-\Pi_{h} u\right\|_{h} \leq \frac{\mu_{b}}{C_{e}}\left\|u-\Pi_{h} u\right\|_{h, *}$. Hence, applying the triangle inequality $\| u-$ $u_{h}\left\|_{h} \leq\right\| u-\Pi_{h} u\left\|_{h, *}+\right\| u_{h}-\Pi_{h} u \|_{h}$, and using the interpolation estimates (21), we can derive the desired estimate (22).

\section{Matrix assembly and decoupling}

Let us assume that the spatial domain $\Omega$ is described as the image of a regular B-spline parameterization

$$
F:(0,1)^{d} \longrightarrow \Omega
$$

We obtain a parameterization $\boldsymbol{\Phi}: \hat{Q} \longrightarrow Q$ of the space-time cylinder $Q$, see (9), by lifting $F$ linearly, i.e.,

$$
\boldsymbol{\Phi}\left(\hat{x}_{1}, \ldots, \hat{x}_{d}, \hat{t}\right)=\left(F\left(\hat{x}_{1}, \ldots, \hat{x}_{d}\right), \hat{t} T\right)
$$

where instead of $\hat{x}_{d+1}$ we use $\hat{t}$ to denote the time variable.

In this section, we exploit the tensor product structure of the spline space to vastly improve the computational complexity of computing the discrete bilinear form $a_{h}(\cdot, \cdot)$, see $(14)$. For the general case of varying coefficient $\rho$, we apply the decoupling technique presented in [17] in order to fully decouple the assembly. Note, that in the case of a constant diffusion coefficient, i.e., $\rho \equiv 1$, the decoupling of the integration in time and in space follows naturally from the form of (2) and (24). In the sequel we present first the case $\rho \equiv 1$.

\subsection{Fast assembly for constant diffusion coefficients}

In this case, we can exploit the tensor product structure of the B-Spline basis and the corresponding structure of the parameterization (24) directly, in order to separate the integration in space and time. For assembling the system matrix produced in (14), we need to compute the bilinear form $a_{h}(\cdot, \cdot)$, on the basis functions of the discrete space. Using the parameterization (24) we can transform each appearing integral on the $d+1$-dimensional space-time domain $Q$ to the parametric domain $\hat{Q}=(0,1)^{d+1}$. As a consequence of the B-Spline basis' tensor-product structure, the resulting $(d+1)$-variate integrals split into a product of a $d$-variate integral over the spatial parametric domain and a univariate integral over the unit interval.

Let $i=\left(i_{1}, \ldots, i_{d}, i_{d+1}\right)$ and $j=\left(j_{1}, \ldots, j_{d}, j_{d+1}\right)$ be two multi-indices. We consider each of the four terms of $a_{h}\left(B_{i}, B_{j}\right)$ separately.

For the first term, the transformation of the integral yields

$$
\begin{aligned}
& \int_{Q} \partial_{t} B_{i} B_{j} d x d t=\int_{\hat{Q}}\left|\operatorname{det} J_{F}\right| \partial_{\hat{t}} \hat{B}_{i} \hat{B}_{j} d \hat{x} d \hat{t} \\
& =\int_{(0,1)^{d}}\left|\operatorname{det} J_{F}\right| \hat{B}_{i_{1} \ldots i_{d}} \hat{B}_{j_{1} \ldots j_{d}} d \hat{x} \cdot \int_{0}^{1} \partial_{\hat{t}} \hat{B}_{i_{d+1}} \hat{B}_{j_{d+1}} d \hat{t}
\end{aligned}
$$


where $J_{F}$ is the Jacobian of the spatial geometric mapping $F$.

For the second term we arrive at

$$
\begin{aligned}
& \theta h \int_{Q} \partial_{t} B_{i} \partial_{t} B_{j} d x d t=\theta \frac{h}{T} \int_{\hat{Q}}\left|\operatorname{det} J_{F}\right| \partial_{\hat{t}} \hat{B}_{i} \partial_{\hat{t}} \hat{B}_{j} d \hat{x} d \hat{t} \\
& =\theta \frac{h}{T} \int_{(0,1)^{d}}\left|\operatorname{det} J_{F}\right| \hat{B}_{i_{1} \ldots i_{d}} \hat{B}_{j_{1} \ldots j_{d}} d \hat{x} \cdot \int_{0}^{1} \partial_{\hat{t}} \hat{B}_{i_{d+1}} \partial_{\hat{t}} \hat{B}_{j_{d+1}} d \hat{t} .
\end{aligned}
$$

Transforming the third term leads to

$$
\begin{aligned}
& \int_{Q} \nabla_{x} B_{i} \nabla_{x} B_{j} d x d t=T \int_{\hat{Q}}\left|\operatorname{det} J_{F}\right| \nabla_{\hat{x}} \hat{B}_{i} J_{F}^{-1} J_{F}^{-\top} \nabla_{\hat{x}} \hat{B}_{j} d \hat{x} d \hat{t} \\
& =T \int_{(0,1)^{d}}\left|\operatorname{det} J_{F}\right| \nabla_{\hat{x}} \hat{B}_{i_{1} \ldots i_{d}} J_{F}^{-1} J_{F}^{-\top} \nabla_{\hat{x}} \hat{B}_{j} d \hat{x} \cdot \int_{0}^{1} \hat{B}_{i_{d+1}} \hat{B}_{j_{d+1}} d \hat{t} .
\end{aligned}
$$

Finally, the fourth integral becomes

$$
\begin{aligned}
& \theta h \int_{Q} \nabla_{x} B_{i} \cdot \nabla_{x} \partial_{t} B_{j} d x d t=\theta h \int_{\hat{Q}}\left|\operatorname{det} J_{F}\right| \nabla_{\hat{x}} \hat{B}_{i} J_{F}^{-1} J_{F}^{-\top} \nabla_{\hat{x}} \hat{B}_{j} d \hat{x} d \hat{t} \\
& =\theta h \int_{(0,1)^{d}}\left|\operatorname{det} J_{F}\right| \nabla_{\hat{x}} \hat{B}_{i_{1} \ldots i_{d}} J_{F}^{-1} J_{F}^{-\top} \nabla_{\hat{x}} \hat{B}_{j_{1} \ldots j_{d}} d \hat{x} \cdot \int_{0}^{1} \hat{B}_{i_{d+1}} \partial_{\hat{t}} \hat{B}_{j_{d+1}} d \hat{t} .
\end{aligned}
$$

We observe that these representations only consist of entries of the stiffness and mass matrices for space and time, as well as those of the matrix containing the mixed time derivatives.

The decomposition of the integrals implies that we can write the system matrix $\left(K_{i j}\right)=\left(a_{h}\left(B_{j}, B_{i}\right)\right)$ as the sum of Kronecker products

$$
K=X_{1} \otimes\left(Y_{1}+Y_{2}\right)+X_{2} \otimes\left(Y_{3}+Y_{1}^{\top}\right),
$$

where $X_{k}$ are $n_{1} \cdots n_{d} \times n_{1} \cdots n_{d}$-matrices containing the d-variate integrals given in (25)-(28) and $Y_{k}$ are $n_{d+1} \times n_{d+1}$-matrices containing the corresponding univariate integrals. This representation (29) is also called the Kronecker format. We define the Kronecker rank of a matrix to be the number of summands in the Kronecker format, that is, in our case the Kronecker rank of $K$ is 2 .

\subsection{Fast assembly for space-time dependent diffusion}

Next we consider the case where the diffusion coefficient $\rho$ is a smooth function depending on both $x$ and $t$. Now, the terms (27) and (28) no longer decompose directly as in the previous case. In order to decouple integration in space and time in this case, we use the partial tensor decomposition method presented in [17] to decompose the parametric diffusion coefficient $\hat{\rho}(\hat{x}, \hat{t})=(\rho \circ \boldsymbol{\Phi})(\hat{x}, \hat{t})$ into $d$-variate and univariate functions by projecting into a spline space and computing the singular value decomposition of the coefficient tensor. This results in an approximation

$$
\hat{\rho}(\hat{x}, \hat{t}) \approx \sum_{r=1}^{R} \mathcal{U}_{r}(\hat{x}) \mathcal{V}_{r}(\hat{t}),
$$

where $\mathcal{U}$ and $\mathcal{V}$ are $d$-variate and univariate spline functions respectively and $R$ is the smallest rank, such that a given error tolerance is satisfied. 
This decomposition of $\hat{\rho}$ leads to a decomposition of the $(d+1)$-variate integrals. In particular, the third term becomes

$$
\begin{gathered}
\int_{Q} \rho(x, t) \nabla_{x} B_{i} \nabla_{x} B_{j} d x d t \approx \\
T \sum_{r=1}^{R} \int_{(0,1)^{d}}\left|\operatorname{det} J_{F}\right| \mathcal{U}_{r}(\hat{x}) \nabla_{\hat{x}} \hat{B}_{i_{1} \ldots i_{d}} J_{F}^{-1} J_{F}^{-\top} \nabla_{\hat{x}} \hat{B}_{j} d \hat{x} \int_{0}^{1} \mathcal{V}_{r}(\hat{t}) \hat{B}_{i_{d+1}} \hat{B}_{j_{d+1}} d \hat{t}
\end{gathered}
$$

and the fourth term becomes

$$
\begin{aligned}
& \theta h \int_{Q} \rho(x, t) \nabla_{x} B_{i} \cdot \nabla_{x} \partial_{t} B_{j} d x d t \approx \\
& \theta h \sum_{r=1}^{R} \int_{(0,1)^{d}}\left|\operatorname{det} J_{F}\right| \mathcal{U}_{r}(\hat{x}) \nabla_{\hat{x}} \hat{B}_{i_{1} \ldots i_{d}} J_{F}^{-1} J_{F}^{-\top} \nabla_{\hat{x}} \hat{B}_{j_{1} \ldots j_{d}} d \hat{x} \int_{0}^{1} \mathcal{V}_{r}(\hat{t}) \hat{B}_{i_{d+1}} \partial_{\hat{t}} \hat{B}_{j_{d+1}} d \hat{t} .
\end{aligned}
$$

We arrive at a Kronecker format representation of rank $R+1$ of the system matrix:

$$
K \approx X_{1} \otimes\left(Y_{1}+Y_{2}\right)+\sum_{r=1}^{R} U^{r} \otimes\left(V_{1}^{r}+V_{2}^{r}\right),
$$

where the matrices $\left.X_{1}, Y_{1}, Y_{2}\right)$ are defined as in (29) and $U^{r}, V_{1}^{r}, V_{2}^{r}$ are the resulting matrices in (30).

If the diffusion coefficient is matrix-valued, let us say $\rho_{p q}$, then the same method can be applied by decomposing each of the components of the matrix. In this case the rank $R$ in the Kronecker format is the total rank, i.e. the sum of the ranks of all components.

\subsection{Computational complexity}

In the following complexity analysis, we assume that the degrees of freedom and polynomial degrees in each $\hat{x}_{k}, k=1, \ldots, d+1$ direction are the same, i.e., $n=n_{1}=\ldots=n_{d+1}$ and $p=p_{1}=\ldots=p_{d+1}$.

The complexity of assembling the system matrix is bounded from below by the number of its non-zeros, which is $O\left(n^{d+1} p^{d+1}\right)$. The classical assembly method using element-wise Gauss quadrature rules has complexity $O\left(n^{d+1} p^{3(d+1)}\right)$.

In the proposed method we compute $d$-variate and univariate integrals by element-wise Gauss quadrature exploiting the decomposition of the integrals. The complexity of computing each matrix $X_{1}, U^{r}$ is thus $O\left(n^{d} p^{3 d}\right)$ while the complexity of computing the matrices $Y_{1}, Y_{2}, V^{r}$ is clearly dominated by this. Thus, the complexity of the quadrature step is

$$
O\left(R n^{d} p^{3 d}\right)
$$

Generating the global matrix $K$ by computing the Kronecker product (31) then costs

$$
O\left(R n^{d+1} p^{d+1}\right) \text {. }
$$

Depending on the dimension, the overall complexity is either dominated by the $d$-variate quadrature or by the sum of Kronecker products. Since usually $n \gg p$, for $d=2$ the complexity of the sum of Kronecker products (31) is dominating. For $d=3$, the complexity of the quadrature step dominates. For details on the complexity see [17].

\section{Numerical examples}

In this section we perform our experiments on a single patch. For a multi-patch domain one can apply continuous or discontinuous discretization techniques and ultimately treat the problem patch-wise. Hence 
the methods developed in this work can be applied for handling the resulting local problems. Typically, the global system matrix for a multi-patch discretization has sparse block structure. Each block has either tensor-product structure similar to (31), or is a very sparse block coming from interface coupling, which can be done in different ways. For instance, the isogeometric tearing and interconnecting (IETI) method from [9] can be applied if the multi-patch discretization is continuous. Otherwise a discontinuous Galerkin approach can be used as is described in [7] for constant coefficient $\rho=1$. The method produces a block-bidiagonal system matrix which can be solved sequentially.

Example 1, $Q \subset \mathbb{R}^{2+1}$.

For our first numerical example, the space-time domain $Q$ is the quarter annulus in space prolongated into the unit interval in time. We consider the function

$$
u\left(x_{1}, x_{2}, t\right)=(\cos (2 \pi(x-y))-\cos (2 \pi(x+y))) \sin (2 \pi t)
$$

to be the exact solution of the problem. The right-hand-side $f$, the boundary data and the initial data are computed accordingly. Besides the system matrix $K$, we also compute the load vector using a partial low-rank approximation of the right-hand-side as presented in [17]. The parameter $\theta$ is set to 1 . The method
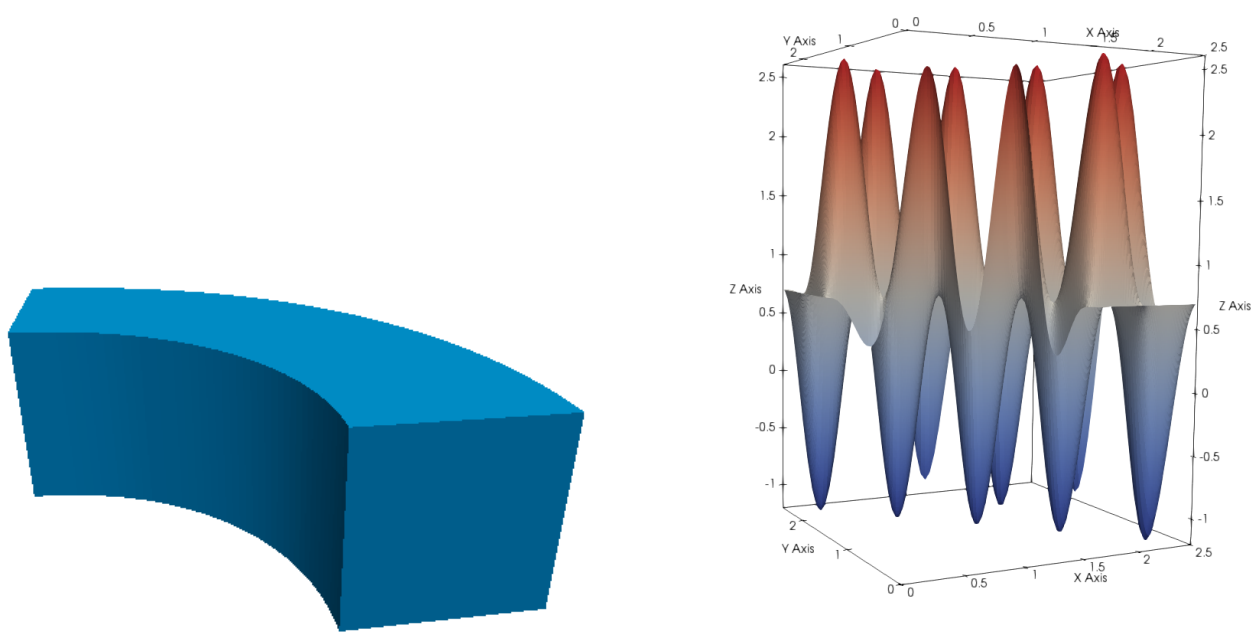

Fig. 2: The space-time domain for $d=2$ and a time slice of the exact solution at $t=0.7$. On the left, the spatial domain is an annulus and the time direction is parallel to the $z$-axis.

was implemented using the $\mathrm{G}+\mathrm{Smo} \mathrm{C}++$ library $[12,16]$. The assembly was performed on a single $2 \mathrm{GHz}$ processor, using B-spline basis functions of degrees 3 and 4.

For solving the linear system, a parallel GMRES solver from Trilinos [6] is used with tolerance set to $10^{-8}$ and Krylov subspace dimension 200. We used a parallel domain decomposition preconditioner (AZ_dom_decomp) and a direct solver (incomplete LU) in each processor/sub-problem.

Table 1 shows the error convergence as well as the computation times for assembling the matrix in the case of a constant diffusion coefficient $\rho \equiv 1$. We observe that the convergence rates are in agreement with the theoretical predicted rates in Theorem 5 . We also remark, that the assembly times given in last column are significantly low compared to the size of the system.

Next, we study the behavior of the method when we have a space-time dependent diffusion coefficient. We consider again the same exact solution given in (32), but the diffusion coefficient is defined as

$$
\rho\left(x_{1}, x_{2}, t\right)=\left(x_{1}-4\right)^{2}\left(x_{2}-4\right)^{2}(t-4)^{2}+\left(x_{1}-4\right)^{4}\left(x_{2}-4\right)^{4}(t-4)^{4} .
$$




\begin{tabular}{l||l|r||l|c|r}
\hline & $\mathbf{h}$ & \#DOF & error & error rate & assembly time \\
\hline \multirow{5}{*}{$p=3$} & 0.25 & 343 & 8.30949 & & $0.0061 \mathrm{~s}$ \\
& 0.125 & 1331 & 5.09944 & 0.70 & $0.0052 \mathrm{~s}$ \\
& 0.0625 & 6859 & 0.492096 & 3.37 & $0.0259 \mathrm{~s}$ \\
& 0.03125 & 42875 & 0.034556 & 3.83 & $0.0926 \mathrm{~s}$ \\
& 0.01562 & 300763 & 0.00392612 & 3.14 & $0.374241 \mathrm{~s}$ \\
\hline \multirow{5}{*}{$p=4$} & 0.25 & 512 & 7.97706 & & $0.0111 \mathrm{~s}$ \\
& 0.125 & 1728 & 5.78954 & 0.46 & $0.0613 \mathrm{~s}$ \\
& 0.0625 & 8000 & 0.2335 & 4.63 & $0.4567 \mathrm{~s}$ \\
& 0.03125 & 46656 & 0.00601485 & 5.28 & $1.9032 \mathrm{~s}$ \\
& 0.01562 & 314432 & 0.000286093 & 4.39 & $11.2130 \mathrm{~s}$ \\
\hline
\end{tabular}

Table 1: 3D experiment on the example space-time domain (see Fig. 2) with a constant diffusion coefficient.

As tolerance for the projection and truncation error in the low-rank approximation we chose $\epsilon=10^{-6}$ which results in an approximation of rank 2 .

\begin{tabular}{l||l|r||l|r|r}
\hline & $\mathbf{h}$ & \#DOF & error & error rate & assembly time \\
\hline \multirow{5}{*}{$p=3$} & 0.25 & 343 & 8.40222 & & $0.0425 \mathrm{~s}$ \\
& 0.125 & 1331 & 5.11743 & 0.72 & $0.0250 \mathrm{~s}$ \\
& 0.0625 & 6859 & 0.492269 & 3.38 & $0.1312 \mathrm{~s}$ \\
& 0.03125 & 42875 & 0.034559 & 3.83 & $0.9555 \mathrm{~s}$ \\
& 0.01562 & 300763 & 0.00366286 & 3.24 & $6.2758 \mathrm{~s}$ \\
\hline \multirow{5}{*}{$p=4$} & 0.25 & 512 & 8.03895 & & $0.01667 \mathrm{~s}$ \\
& 0.125 & 1728 & 5.81114 & 0.47 & $0.1474 \mathrm{~s}$ \\
& 0.0625 & 8000 & 0.233569 & 4.64 & $0.2993 \mathrm{~s}$ \\
& 0.03125 & 46656 & 0.00601493 & 5.28 & $1.9955 \mathrm{~s}$ \\
& 0.01562 & 314432 & 0.000293 & 4.36 & $16.8282 \mathrm{~s}$ \\
\hline
\end{tabular}

Table 2: 3D experiment on the example space-time domain (see Fig. 2) with a space-time dependent diffusion coefficient (2).

For the cases of $p \geq 5$, we only show the computation times of the assembly, since the resulting systems are not only non-symmetric but also quite large and dense, therefore rather hard to work with. Figure 3 shows the dependence of the computation times on the number of degrees of freedom for the partial tensor decomposition method as well as for the classical element-wise Gauss quadrature. It can be seen in the presented computation times that the assembly method using partial low-rank tensor approximation is very efficient. In particular, it outperforms a classical element-wise Gauss rule approach by far. We refer to [17] for further experimental comparisons between the classical element-wise Gauss quadrature and the low-rank partial tensor decomposition method, for the stiffness matrix.

For the cases of $p \geq 5$, we only show the computation times of the assembly, since the resulting systems are not only non-symmetric but also quite large and dense, therefore very hard to solve. Figure 3 shows the dependence of the computation times on the number of degrees of freedom for the 3D problem. As it can be seen in the presented computation times, the assembly method using partial low-rank tensor approximation is very efficient. In particular, it outperforms a classical element-wise Gauss rule approach by far. We refer to [17] for an experimental comparison between the classical element-wise Gauss quadrature and the low-rank partial tensor decomposition method, for the stiffness matrix.

In some cases it can be beneficial to avoid evaluating the sum of Kronecker products in (31) by using the Kronecker format representation directly. Since matrix-vector multiplication can be implemented easily for a matrix in this format, it can be used for solving the system iteratively. The main advantage is the 
reduction of the needed memory for storing the system matrix which allows us to assemble up to a very large number of degrees of freedom. The assembly is also very fast, since we only have to compute bivariate and univariate integrals. Figure 4 shows the computation times for the bivariate and univariate integrals in the matrices of the right hand side in (31). The maximum number of degrees of freedom that were computed in this experiment are over 136 million for $p=3$.

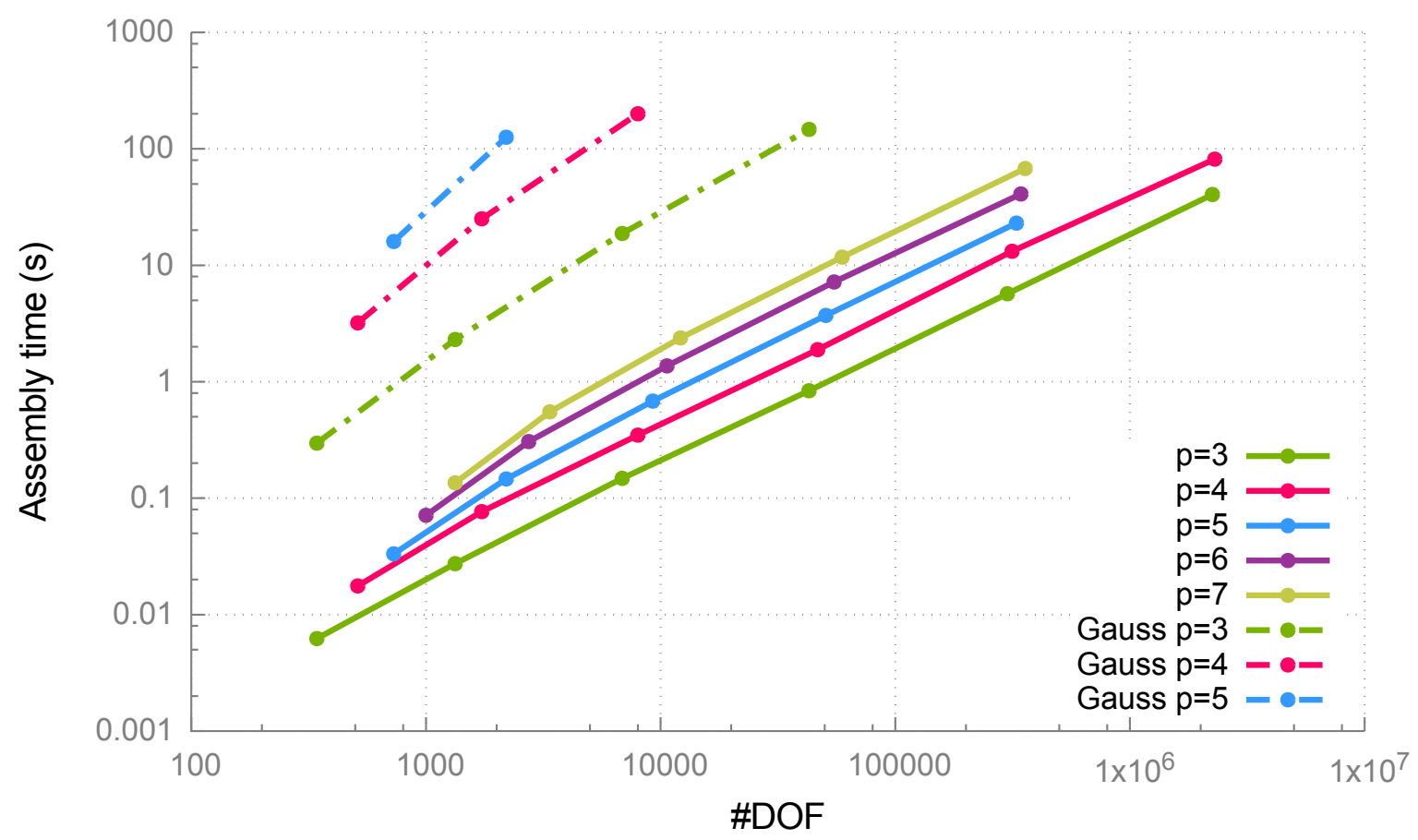

Fig. 3: Computation times for assembling the global system matrix on the example domain (Fig. 2) for the spacetime dependent diffusion coefficient (2).

Example 2, $Q \subset \mathbb{R}^{3+1}$.

In this example the space-time cylinder $Q$ is the product of a volumetric shell shape (see Fig. 5) in space and the unit interval in time. The diffusion coefficient is chosen to be

$$
\begin{aligned}
\rho\left(x_{1}, x_{2}, x_{3}, t\right) & =\left(x_{1}-4\right)^{2}\left(x_{2}-4\right)^{2}\left(x_{3}-4\right)^{2}(t-4)^{2}+ \\
& +\left(x_{1}-4\right)^{4}\left(x_{2}-4\right)^{4}\left(x_{3}-4\right)^{4}(t-4)^{4} .
\end{aligned}
$$

We focus on the computation times for matrix assembly. Figure 6 shows the dependence of the assembly time on the number of degrees of freedom for the $\rho\left(x_{1}, x_{2}, x_{3}, t\right)$ given in (34).

We note that in the four-dimensional case the overall complexity is no longer dominated by the sum of Kronecker products (31) but by the trivariate quadrature. For this reason, the computation of the matrix in Kronecker format is no longer significantly faster than the computation of the global matrix by performing the sum of Kronecker products in (31). However, the advantages stemming from the reduction in memory still apply to the four-dimensional case and we can assemble up to many more degrees of freedom using the same amount of memory.

Figure 7 shows the computation times for the system matrix in Kronecker format. They are dominated by the trivariate spatial integrals. For $p=2$ we assembled for a maximum number of about 19 million degrees of freedom. Finally, we observe one more time that the partial tensor decomposition method leads to a large speed-up of the computation compared to the classical element-wise Gauss quadrature. 


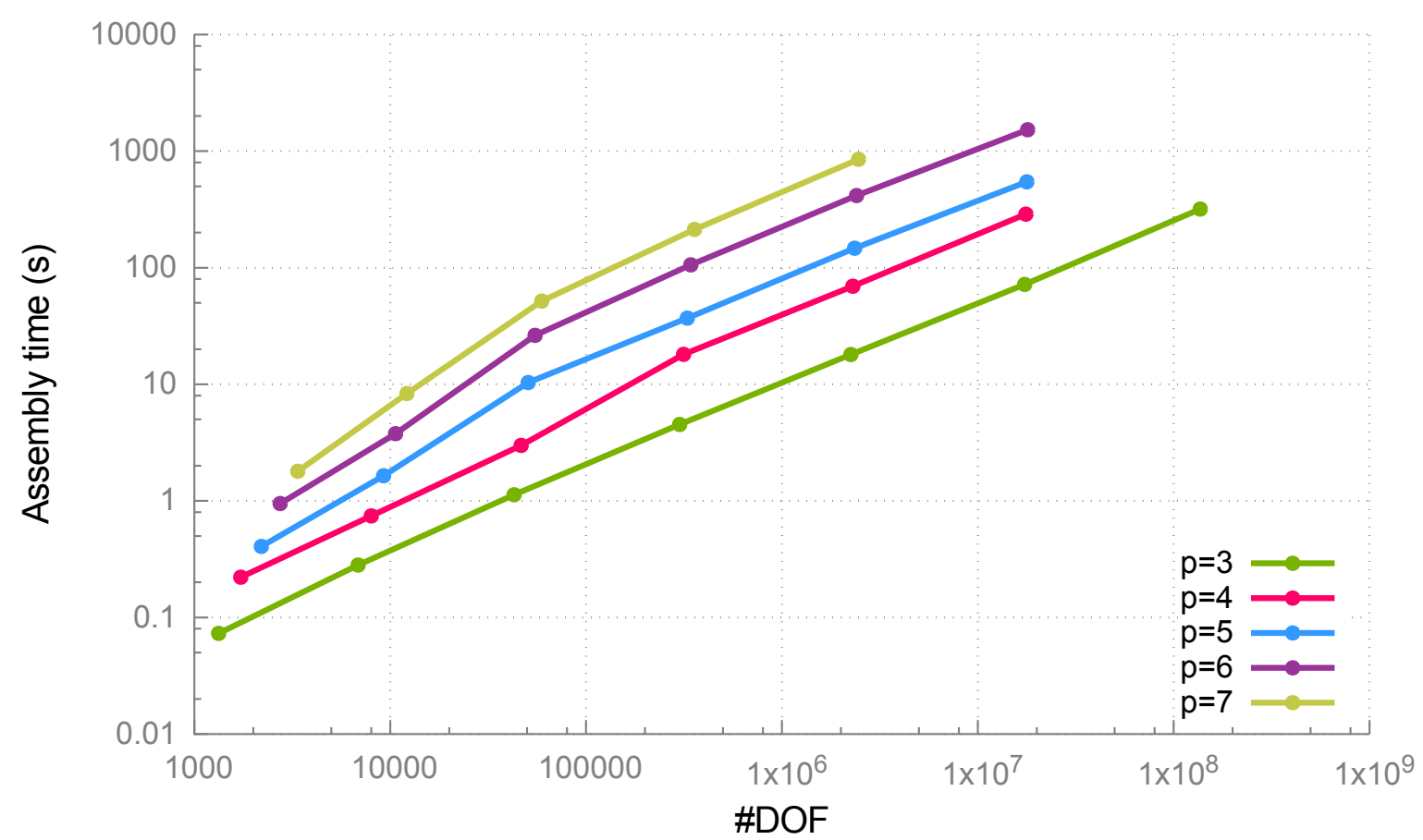

Fig. 4: Computation times for assembling the Kronecker format on the example domain (Fig. 2) for the space-time dependent diffusion coefficient (2).

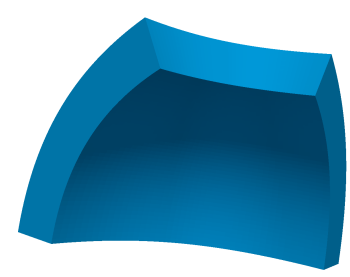

Fig. 5: The volumetric shell shape of Example 2.

\section{Conclusions}

We presented and analyzed a space-time $\operatorname{IgA}$ scheme for linear parabolic problems with varying coefficients. We employed low-rank approximation techniques to speed-up the computation of the discrete operator. In this way we were able to compute with several millions of degrees of freedom. However, a standing challenge is the solution of the linear system. Indeed, this system is non-symmetric and with a rapidly increasing bandwidth with respect to the polynomial degree. The GMRES solver that we used, combined with domain decomposition and ILU preconditioner performed reasonably good for a moderate number of degrees of freedom. Another challenging task is the extension of this approach to problems with discontinuous diffusion coefficients. In this case, the space-time cylinder $Q$ could be described as a multi-patch space-time domain (cf. [12]) compatible with the discontinuities of the diffusion coefficient. The low-rank approximation would be applied patch-wise and appropriate discontinuous Galerkin techniques, i.e., numerical fluxes, would be needed for coupling the local patch-wise problems.

\section{Acknowledgment}

This work was supported by the Austrian Science Fund (FWF) under the grant NFN S117. 


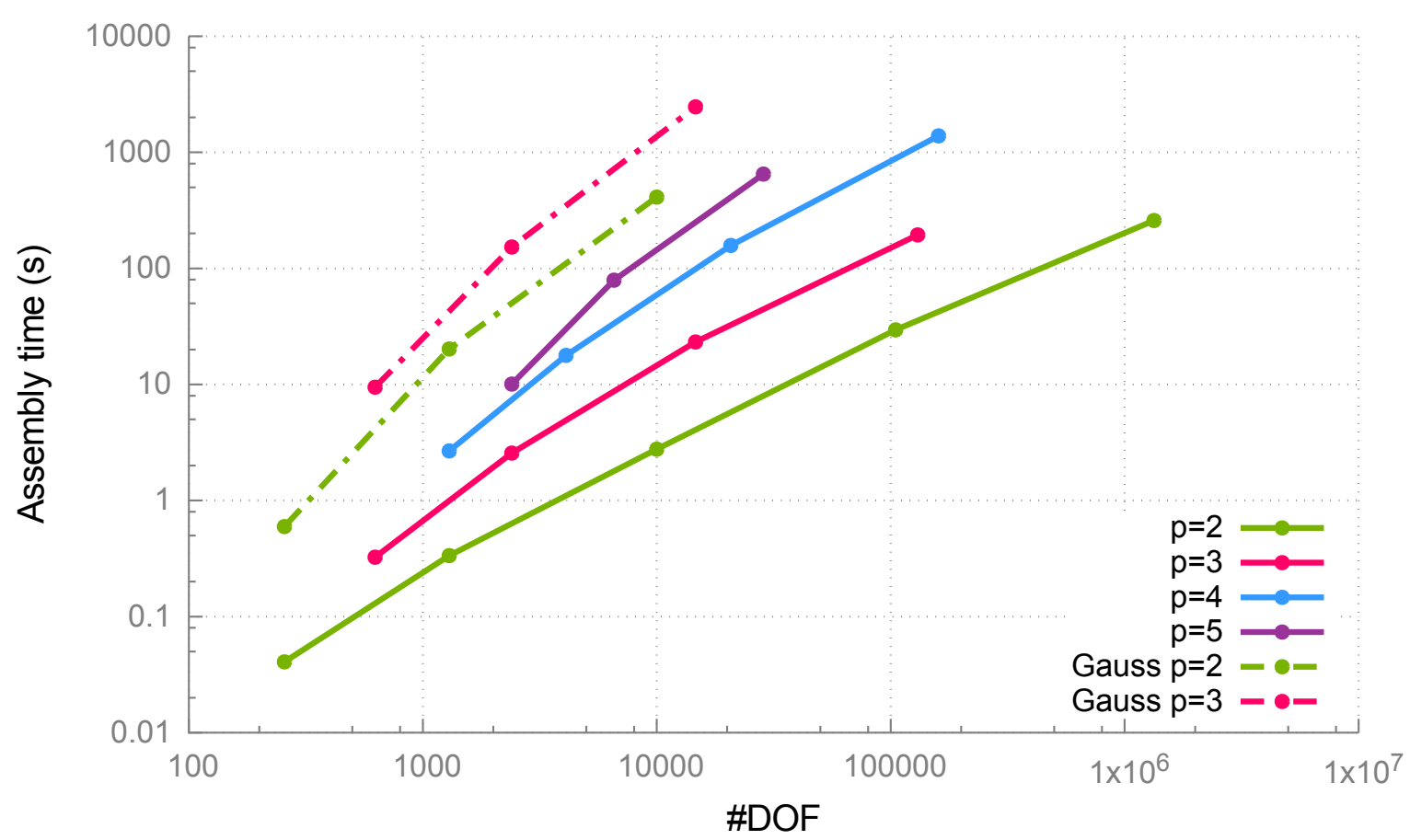

Fig. 6: Computation times for assembling the global system matrix on the four-dimensional space-time domain (with spatial part as in Fig. 5) for the space-time dependent diffusion coefficient (34).

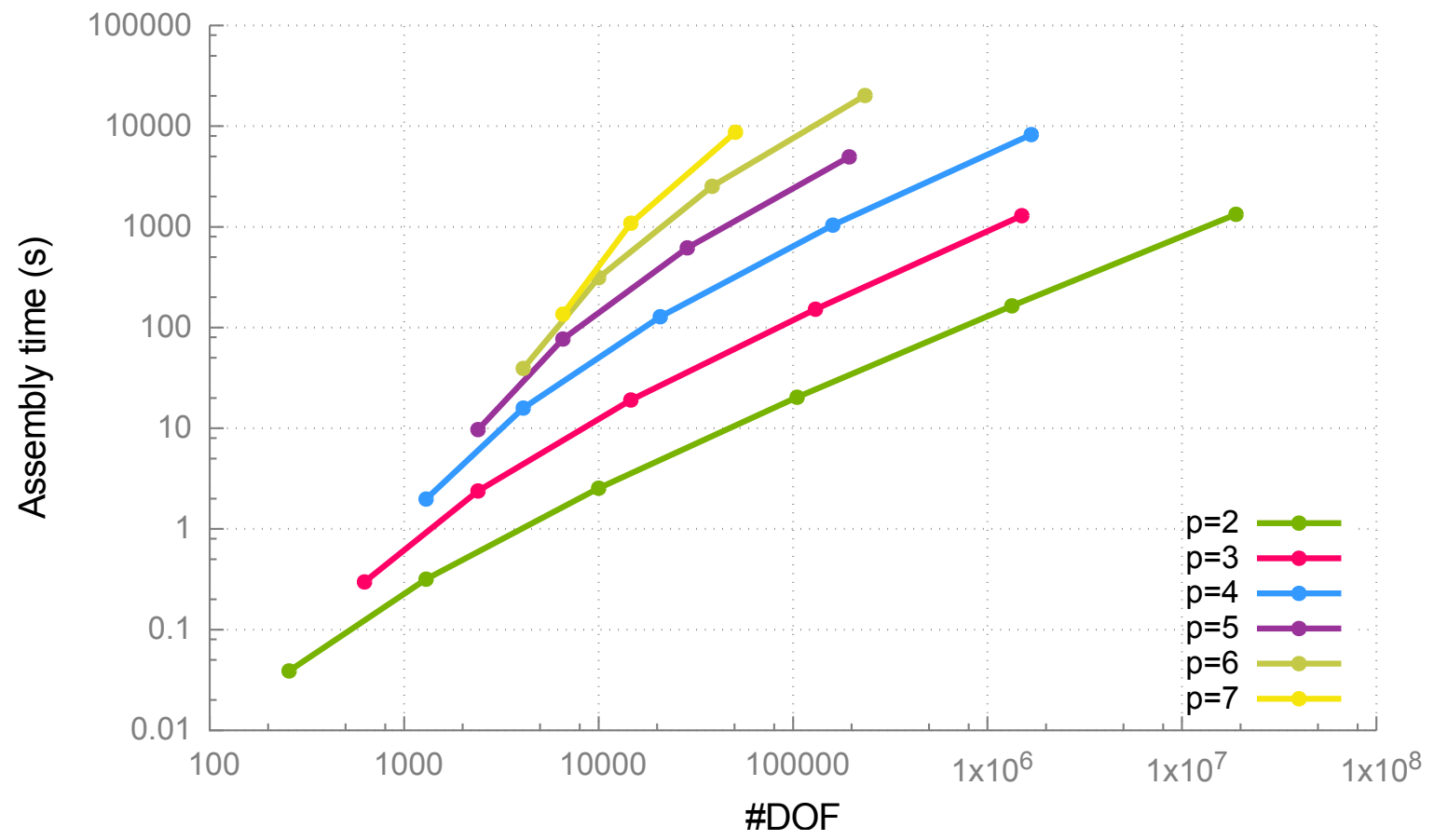

Fig. 7: Computation times for assembling the Kronecker format on the four-dimensional space-time domain (with spatial part as in Fig. 5) for the space-time dependent diffusion coefficient (34).

\section{References}

[1] J. A. Cotrell, T.J.R. Hughes, and Y. Bazilevs. Isogeometric Analysis, Toward Integration of CAD and FEA. John Wiley and Sons, 2009. 
[2] L. Beirão da Veiga, A. Buffa, G. Sangalli, and R. Vázquez. Mathematical analysis of variational isogeometric methods. Acta Numerica, 23:157-287, 52014.

[3] C. De-Boor. A Practical Guide to Splines, volume 27 of Applied Mathematical Science. Springer, New York, revised edition edition, 2001.

[4] S. Dolgov and B.N. Khoromskij. Simultaneous state-time approximation of the chemical master equation using tensor product formats. Numerical Linear Algebra with Applications, 22:197-219, 2015.

[5] S. V. Dolgov, B. N. Khoromskij, and I. V. Oseledets. Fast solution of parabolic problems in the tensor train/quantized tensor train format with initial application to the fokker-planck equation. SIAM Journal on Scientific Computing, 34(6):A3016-A3038, 2012.

[6] M. Heroux et al. An overview of trilinos. Technical Report SAND2003-2927, Sandia National Laboratories, 2003.

[7] C. Hofer, U. Langer, M. Neumüller, and I.Toulopoulos. Time-multipatch discontinuous Galerkin space-time isogeometric analysis of parabolic evolution problems. RICAM Report No. 2017-26, www.ricam.oeaw.ac.at/publications/ricam-reports/?year=2017, 2017.

[8] T.J.R. Hughes, J. A. Cottrell, and Y. Bazilevs. Isogeometric analysis : CAD, finite elements, NURBS, exact geometry and mesh refinement. Computer Methods in Applied Mechanics and Engineering, 194:4135-4195, 2005.

[9] S. Kleiss, C. Pechstein, B. Jüttler, and Satyendra Tomar. IETI - Isogeometric Tearing and Interconnecting. Computer Methods in Applied Mechanics and Engineering, 247-248:201 - 215, 2012.

[10] O. A. Ladyzhenskaya. The Boundary Value Problems of Mathematical Physics. Springer, New York, 1985.

[11] O. A. Ladyzhenskaya, V. A. Solonnikov, and N. N. Uraltseva. Linear and Quasilinear Equations of Parabolic Type. AMS, Providence, RI, 1968.

[12] U. Langer, A. Mantzaflaris, St. E. Moore, and I. Toulopoulos. Multipatch discontinuous Galerkin isogeometric analysis. In B. Jüttler and B. Simeon, editors, Isogeometric Analysis and Applications IGAA 2014, volume 107 of Lecture Notes in Computer Science, pages 1-32, Heidelberg, 2015. Springer.

[13] U. Langer, S. Moore, and M. Neumüller. Space-time isogeometric analysis of parabolic evolution equations. Computer Methods in Applied Mechanics and Engineering, 306:342-363, 2016.

[14] A. Mantzaflaris, B. Jüttler, B. Khoromskij, and U. Langer. Matrix generation in isogeometric analysis by low rank tensor approximation. In Curves and Surfaces: 8th International Conference, Paris, France, June 12-18, 2014, Revised Selected Papers, pages 321-340. Springer, 2015.

[15] A. Mantzaflaris, B. Jüttler, B. Khoromskij, and U. Langer. Low rank tensor methods in Galerkin-based isogeometric analysis. Computer Methods in Applied Mechanics and Engineering, 316:1062-1085, 2017.

[16] A. Mantzaflaris, F. Scholz, and others (see website). G+smo (Geometry plus Simulation modules) v0.8.1. http://gs.jku.at/gismo, 2017.

[17] F. Scholz, A. Mantzaflaris, and B. Jüttler. Partial tensor decomposition for decoupling isogeometric Galerkin discretizations. Computer Methods in Applied Mechanics and Engineering, 336:485-506, 2018. 